\title{
Analysis of In-Space Assembly of Modular Systems
}

\author{
Robert W. Moses ${ }^{*}$, James Van Laak ${ }^{\dagger}$, Spencer L. Johnson ${ }^{\ddagger}$, Trina M. Chytka ${ }^{\S}$,

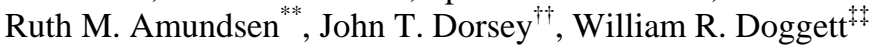 \\ NASA Langley Research Center, Hampton, VA 23681 \\ John D. Reeves ${ }^{\S \S}$ \\ National Institute of Aerospace, Hampton, VA 23666 \\ B. Keith Todd ${ }^{* * *}$ \\ NASA Johnson Space Center, Houston, TX 77058 \\ Damon B. Stambolian ${ }^{\dagger+}$ \\ NASA Kennedy Space Center, KSC, FL 32899 \\ and \\ Rud V. Moe \\ NASA Goddard Space Flight Center, Greenbelt, MD 20771
}

\begin{abstract}
Early system-level life cycle assessments facilitate cost effective optimization of system architectures to enable implementation of both modularity and in-space assembly, two key Exploration Systems Research \& Technology (ESR\&T) Strategic Challenges. Experiences with the International Space Station (ISS) demonstrate that the absence of this rigorous analysis can result in increased cost and operational risk. An effort is underway, called Analysis of In-Space Assembly of Modular Systems, to produce an innovative analytical methodology, including an evolved analysis toolset and proven processes in a collaborative engineering environment, to support the design and evaluation of proposed concepts. The unique aspect of this work is that it will produce the toolset, techniques and initial products to analyze and compare the detailed, life cycle costs and performance of different implementations of modularity for in-space assembly. A multi-Center team consisting of experienced personnel from the Langley Research Center, Johnson Space Center, Kennedy Space Center, and the Goddard Space Flight Center has been formed to bring their resources and experience to this development. At the end of this 30-month effort, the toolset will be ready to support the Exploration Program with an integrated assessment strategy that embodies all life-cycle aspects of the mission from design and manufacturing through operations to enable early and timely selection of an optimum solution among many competing alternatives. Already there are many different designs for crewed missions to the Moon that present competing views of modularity requiring some in-space assembly. The purpose of this paper is to highlight the approach for scoring competing designs.
\end{abstract}

\footnotetext{
* Principal Investigator, Exploration Systems Engineering Branch, MS 472, AIAA Associate Fellow.

${ }^{\dagger}$ Head, Systems Management Office, MS 165.

${ }^{\ddagger}$ Project Manager, Mechanical Systems Branch, Systems Engineering Directorate, MS 468.

$\S$ Operations \& Mission Requirements, Exploration Concepts Branch, MS 365, AIAA Member.

** Aerospace Engineer, Structural and Thermal Systems Branch, MS 431, AIAA Member.

${ }^{\text {t† }}$ Senior Research Engineer, Metals and Thermal Structures Branch, MS 396, AIAA Associate Fellow.

执 Senior Research Engineer, Guidance \& Controls Branch, Research \& Technology Directorate, MS 161.

$\S$ Research Engineer, Research Staff, MS 451, AIAA Member.

${ }^{* * *}$ Robotics and Crew Systems Operations Division, Code DX1, AIAA Senior Member.

${ }^{+\dagger \dagger}$ Human Factors Engineering, Exploration Operations Integrations, Code UB-X.

抹 Senior Research Engineer, Hubble Space Telescope Development Project, Code 442.
} 


\section{Introduction}

$\mathrm{E}$ ARLY system-level life cycle assessments facilitate optimization of system architectures to achieve a costeffective approach to both modularity and in-space assembly, two key Exploration Systems Research \& Technology (ESR\&T) Strategic Challenges. Experiences with the International Space Station (ISS) demonstrate that the absence of this rigorous analysis can result in increased cost and operational risk. The ability to assess those costs and risks driven by modularity and in-space assembly must be available for design selection. Already, different designs for the Crew Exploration Vehicle (CEV) present competing views of modularity requiring some in-space assembly ${ }^{1}$. However, the ability to analyze the many options for comparison in terms of life cycle cost and risk is not available presently.

Analyzing modularity and in-space assembly to assess their impact on mission architecture poses many challenges. For Apollo, a heavy lift launch capability permitted minimal modularity and in-space assembly. That launch capability no longer exists; even if it did, the high degree of human presence envisioned by NASA for the Moon will require far more resources delivered to the surface of the Moon than ever before. Furthermore, placing humans safely on Mars and bringing them safely home pose orders of magnitude more difficulty than previous space accomplishments stemming from Mercury through ISS.

An affordable, sustainable exploration program to Mars and beyond will require technologies that deviate from heritage hardware. Thus, models and analysis will require updating to factor those new technologies into mission architecture. Thus, any analysis approach must be flexible and contain elements that are reliably employed and their results easily understood.

\section{Purpose and Expectations}

The Analysis of In-Space Assembly of Modular Systems effort will produce an analytical methodology to support the design and evaluation of proposed exploration architectures. The methodology will include an evolved analysis toolset and proven processes brought together in a collaborative engineering environment. The toolset, techniques, and initial products will be used to analyze and compare the detailed, life cycle costs and performance of different implementations of modularity for in-space assembly within competing mission architectures, including system design and manufacturing, sustaining engineering, the design robustness for flight operations and consequent requirements for real-time engineering support, crew and flight controller training, and logistics supportability. The tools will provide insight into how the various implementations of modularity affect vehicle mass, power and thermal rejection capability, and vehicle safety through safe haven and other inherent characteristics. Since the combined effects of these implementation decisions can have major impacts on system effectiveness, cost and supportability, this work will provide tools and initial analyses to identify design discriminators and establish the appropriate level of modularity to be sought in the design of Exploration hardware.

A multi-Center team from the Langley Research Center, Johnson Space Center, Kennedy Space Center, and the Goddard Space Flight Center will bring their resources and experience to bear on this work. Leadership and management will be provided by the Langley team, which offers a rich blend of comprehensive systems analysis capability together with a highly experienced systems engineering organization, thus providing technical capability from concept development to design solutions. JSC will provide their extensive expertise in human and robotic extra-vehicular activity, as well as critical operations analysis of system strengths and weaknesses during real-world assembly operations and extended mission operations. KSC will analyze pre-flight processing of modular elements and offer insight into techniques and opportunities for integrated testing prior to launch. GSFC will support the analysis with their expertise on maintenance and repair of the modular systems in flight.

At the end of this 30-month effort, the toolset will be ready to support the Exploration Program with an integrated assessment strategy that embodies all life-cycle aspects of the mission from design and manufacturing through operations to permit early and timely selection of an optimum solution among many competing alternatives. The purpose of this paper is to highlight the approach for scoring competing designs.

\section{Analysis Approach and Focus}

The analysis approach consists of several tasks. First, requirements will be assembled, and evaluation criteria; second, the ensemble of the experts in a collaborative engineering environment to employ analysis tools and intellect for applying the requirements and evaluation criteria. The criteria are applied to the design reference mission from a separate mission architecture study by NASA, and then scoring the competing designs that claim to enable NASA's exploration vision. The streamlining of this analysis capability is also to support simulation based acquisition (SBA). 
This 30-month effort will focus on two key issues: 1) the combined system of systems implications of modular designs and their assembly in space; and 2) the interfaces between modules and their impact on the assembly and detachment phases near Earth and the target destination.

\section{A. Requirements and Evaluation Criteria}

The requirements and evaluation criteria for scoring competing approaches to modularity requiring in-space assembly will include many elements of traditional systems engineering. Life cycle cost will factor strongly in the scoring of competing designs.

The project will include discipline specialists in launch processing from Kennedy Space Center, in-space assembly from Johnson Space Center, and on-orbit servicing from Goddard Space Flight Center. Launch processing requirements include those affecting payload launch preparation, payload launch pad scenarios and sequences, and payload start-up and initial check-out. In-space assembly requires astronaut assembly operations, training, practices, and tools along with interfaces, attachments, mechanisms, and positioning guides. On-orbit servicing also focuses on astronaut workloads and support logistics.

Some additional requirements by the Exploration Systems Mission Directorate are expected to be levied on this project as other projects mature. The NASA-directed requirements will affect the implementation and operations of the analysis capability planned for the Integrated Design Center (IDC) at the Langley Research Center (Figure 1). Working with other integrated design centers in the agency, the IDC tools and operations will provide access to the analysis capabilities for teams supporting coast-to-coast operations as part of NASA's ASCT (Advanced Studies, Concepts, and Tools) Advanced Studies. These requirements may dictate additional software and hardware capabilities for the IDC that would not otherwise be required if all experts were residing in one room. In addition, this project has the responsibility for non-robotic interfaces and their standards because they may impact the design reference architecture. This project will study the heritage and proposed physical interfaces between modules and assess their implications on the life cycle cost of future exploration hardware systems. Furthermore, the analysis capabilities developed for this project may be modified later to accommodate specific requirements on SBA.

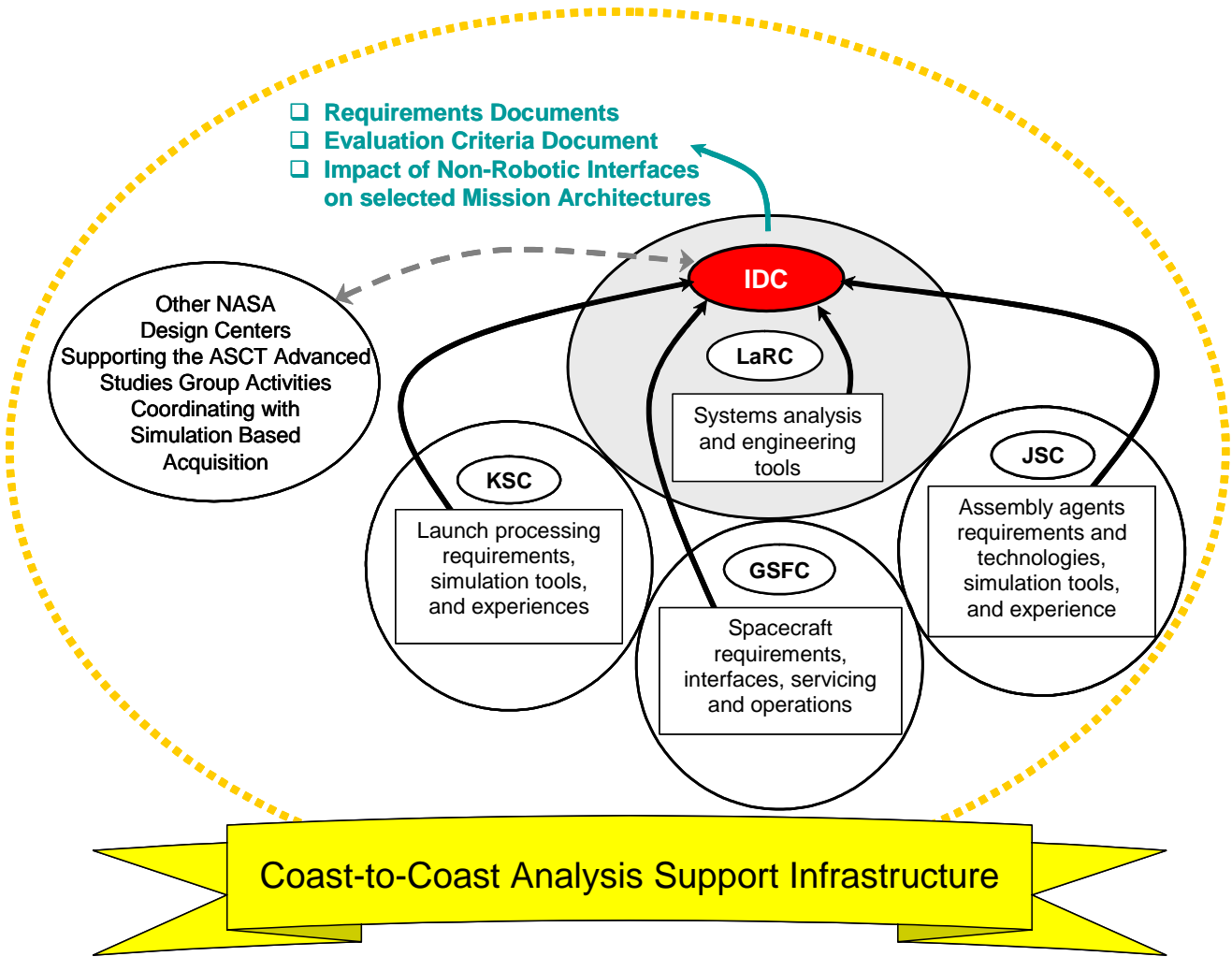

Figure 1. The IDC provides a single design path, simplifies user operations and logistics, and provides for communication among disciplines. 


\section{B. Analysis Capability and Planned Tools}

The purpose of this effort is to develop the tools and the approach to properly analyze the benefits and costs of implementing modularity in a space flight system. The product is the capability to generate a global optimization of the integrated system performance across the entire life cycle of the Exploration Program, with emphasis on the reliability and sustainability parameters that define the most critical performance requirements of exploration systems. Because many disciplines and experiences in space flight across the agency will be required, the best forum for bringing these resources together appears to be a collaborative engineering environment. This type of forum has been implemented successfully for spacecraft missions at JPL and GSFC. The IDC at LaRC provides a similar collaborative forum but with emphasis on systems analysis and systems engineering capabilities (Figure 2). The software tools currently in use at the IDC include commercially available 3-D physics-based simulations. For instance, the IDC has the capability to analyze in-space assembly by combining computer-aided design (CAD) models of modular hardware geometry with multi-satellite trajectory analysis software. At times, new software or hardware tools are incorporated to allow for inclusion of other capabilities into the collaborative engineering environment. As other partners join in the IDC, their software tools will be incorporated into the rich toolset. The tools for this effort currently exist but as components residing at separate locations. They will be integrated into a collaborative engineering toolset and validated against a relevant case.

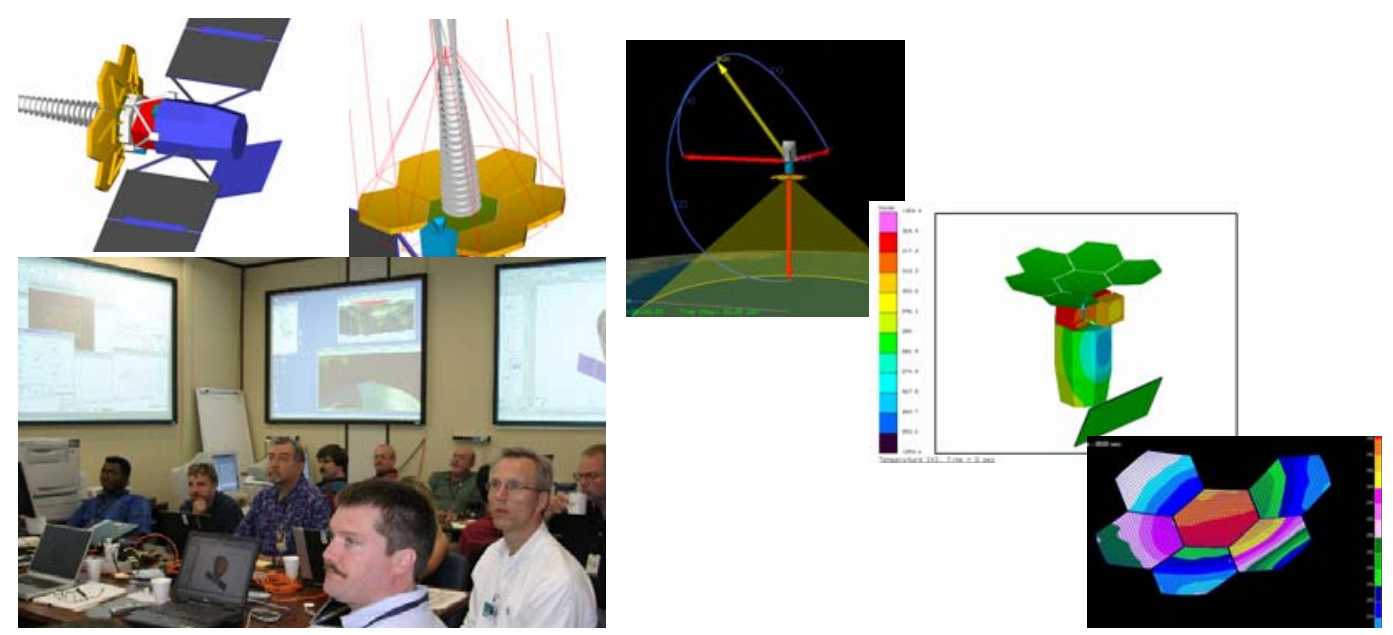

Figure 2. Multiple perspectives of results of a study conducted within the IDC at LaRC provide early insight into design drivers.

Two tools currently in the plans to acquire for the IDC are teleconferencing with other collaborative design centers at JPL and GSFC and discrete event simulation (DES) with integrated life cycle cost features. The former tool will use commercially available items that will require little to no modification to implement within the IDC. For the latter, DES is a numerical computer-based analysis technique that models discrete changes in the state of the system in order to capture the dynamics between various entities and events. DES is a methodology that has gained popularity in the aerospace field due to its ability to dynamically model complex logistical flow paths such as launch vehicle ground preparation operations. A properly developed DES model is capable of capturing a wide variety of model characteristics such as life cycle costs and aggregate vehicle reliabilities. The essence of DES is that it is used to probabilistically study model input assumptions and resulting output metrics in order to better capture real-world uncertainty and variability. One popular commercial DES tool used extensively at Langley Research Center will be added to the IDC when needed by the team during focused activities. An example of the capabilities of DES will be illustrated later in this paper to underscore its importance to this project.

\section{DES and the Analysis of In-Space Assembly of Modular Systems}

The analytical methodology to be developed during this effort includes an evolved analysis toolset that captures all of the disciplines necessary to evaluate proposed concepts. The first phase of the project will determine the disciplines and tools needed to support the analysis. The various discipline tools will be identified and integrated into Langley's IDC, and data standards will be established to ensure that the tools generate results in a standard form (e.g., Standard International units). The collaborative environment may utilize an automated interface or subject matter experts may act as intermediaries between the tools and the rest of the design team. A central tool will bring 
together the output data from the discipline tools in order to generate life cycle scoring. A DES life cycle analysis model, in conjunction with some supporting software such as a spreadsheet utility with embedded macro programs, will facilitate this type of analysis by capturing all of the interactions that take place during a mission cycle such as ground operations, schedule delays, on-orbit assembly operations, and logistical operations beyond LEO. Standardized outputs from the various discipline tools can be brought into the DES model either deterministically or probabilistically to allow evaluation of the scenario and to generate an overall scoring metric or set of metrics. Sensitivity analyses will be performed on the various inputs in order to understand which parameters drive life cycle costs. Sensitivity analysis capability would allow decision makers to see if slight changes in proposed designs could influence which proposal is superior, and thus yield a more robust decision.

NASA has recently begun looking into various ways to implement SBA, an acquisition philosophy that utilizes computer-based simulation techniques to make procurement decisions. DES as a component for the Analysis of InSpace Assembly of Modular Systems project will enhance the Agency’s SBA capabilities.

\section{Existing DES Model for On-Orbit Assembly Analysis}

The current model, designated the Architectural Model, was initially developed to analyze life cycle costs for launch vehicle ground operations for a variety of existing and proposed vehicle concepts, both reusable and expendable. This original model was a high-level simulator of all critical activities that are involved with preparing and recovering from a launch, such as processing reusable components, acquiring expendable components, integration in a dedicated integration facility, launch operations, and recovery and safing activities. The model was later amended to capture on-orbit assembly operations in low earth orbit in order to study modularity impacts of a sample manned lunar mission. Because of this, the model is in line with what the Analysis of In-Space Assembly of Modular Systems effort is trying to accomplish in terms of studying the particulars that go into assembling modular systems on orbit. Added fidelity and a broadening of scope of the Architectural Model will be required during the study in order for the model to act as the life cycle scoring tool for the overall analysis methodology.

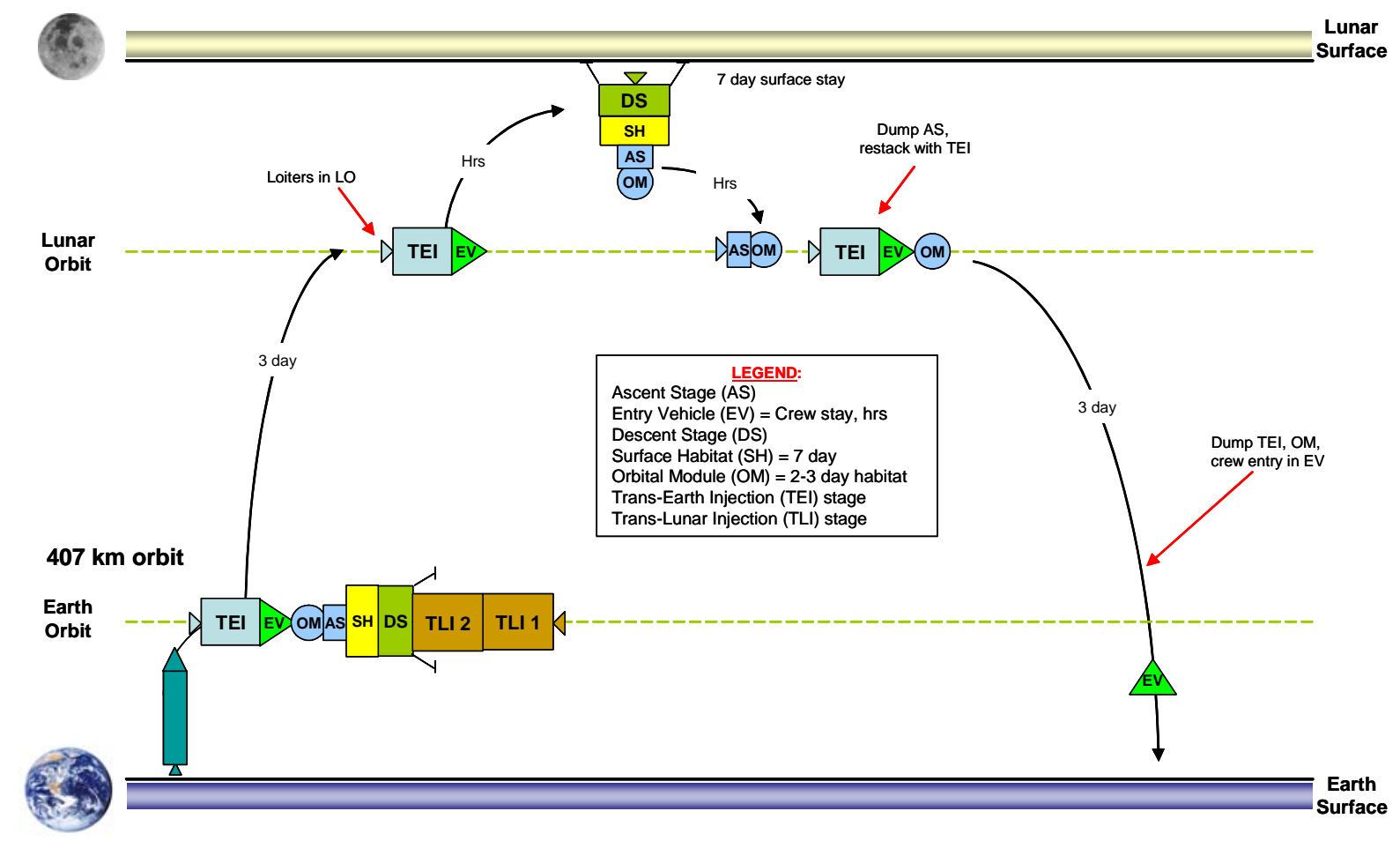

\section{Figure 3. Example reference lunar mission system study showing eight modules with assembly in Earth and Lunar orbits.}

Since the Architectural Model is a top-level analysis tool, various assumptions were made during development in order to narrow the scope of what was being analyzed. For example, the model focuses on one lunar mission at a time, with the definition of a lunar mission consisting of one assembled vehicle being sent on a trans-lunar trajectory 
(figure 3). The model generates both cost and mission success metrics, with cost incorporating just the launch costs associated with the operation of various launch vehicles, and the success metrics being related to successfully getting the modules integrated on orbit and sent on a trans-lunar trajectory before orbit degradation effects become significant (figure 4). Any number of Earth-to-orbit launches can be used in order to deliver vehicle modules to LEO. Each vehicle module delivered to orbit has an associated corresponding automated rendezvous and docking (AR\&D) reliability that allows parametric analysis of the impacts of docking failures. Any sort of docking failure or other failure that prevents a particular vehicle module from integrating with the rest of the modules results in a relaunch, which inherently incurs additional launch costs (figure 5). Any sort of launch vehicle concept can be loaded into the model since there are mechanisms that allow both reusable and expendable process flows to be captured. The model is also capable of capturing launch vehicle costs on either a facility-use basis or a contractual fixed cost per launch.

The Architectural Model is manifest-driven, meaning a launch schedule has to be fed into the model that contains specifics such as target launch dates and payload assignments. There are also two tiers of decision gates embedded in the model, both of which contribute to the resulting cost and success metrics. The upper tier is a lunar mission specific tier that relates to the following: timely ground processing for all launches, successful integration of all modules, and whether or not the assembled vehicle is sent on a trans-lunar trajectory before orbit degradation effects become significant. The second tier of decision points pertains to the individual launches that are specified on the manifest. These decision points include loss of vehicle scenarios during launch, payload delivery failures (payload cannot reach correct orbit), and failure of individual payload modules to integrate. The model is currently set up with no animation features in order to facilitate the rapid execution of a multiple replication run. Visual Basic for Applications (VBA) coding is used via a spreadsheet database tool to load various manifests into the model, execute the model, and to retrieve and tabulate the resulting output metrics in an automated fashion. Because this spreadsheet database tool allows a user to automatically run any number of manifests serially through the model, it lends itself to Design of Experiment (DoE) studies regarding the manifest inputs.

\section{Operational Scenario}

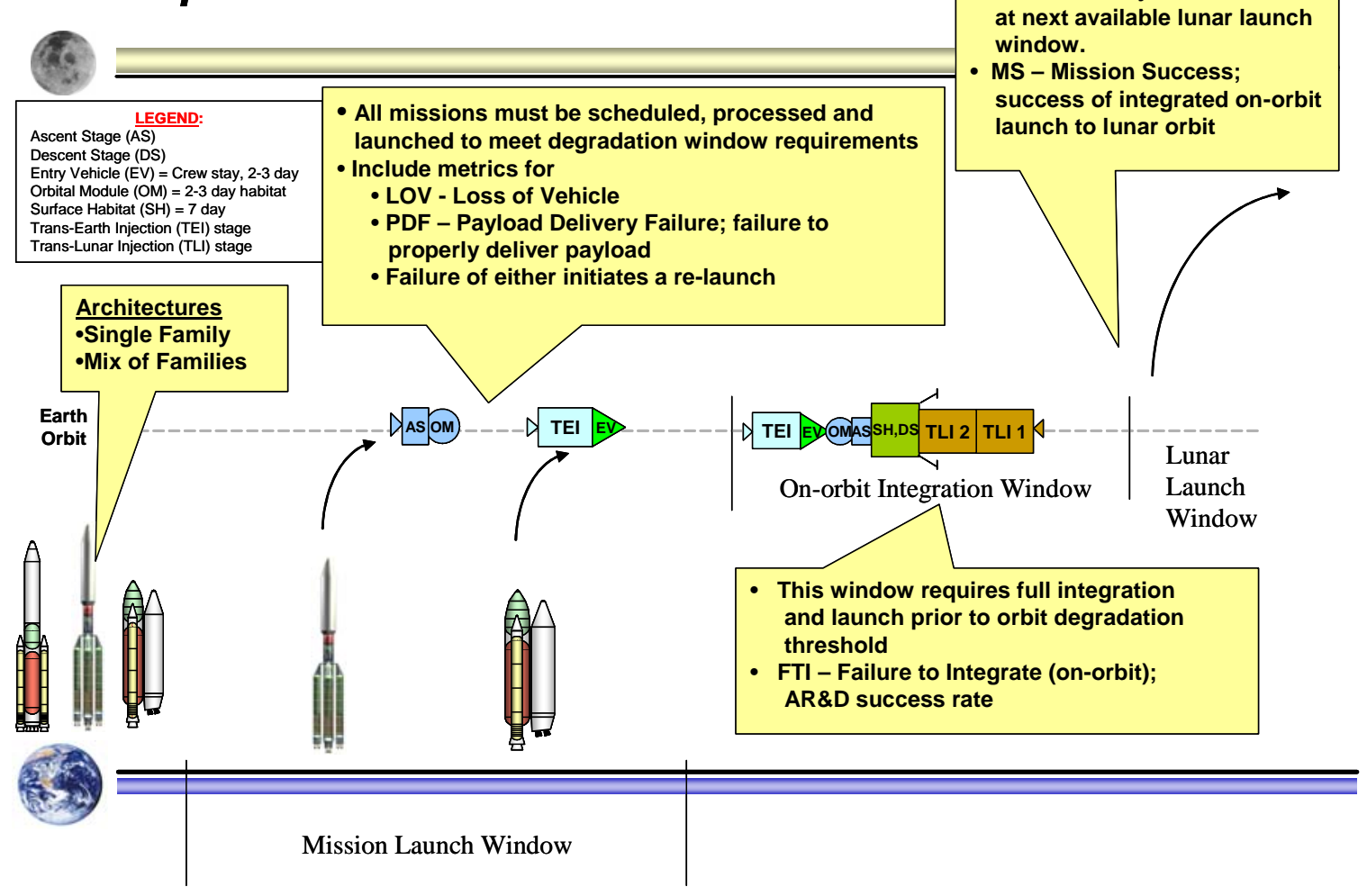

Figure 4. Operational scenario for example reference lunar mission system study. 
Architecture Costs Averaged

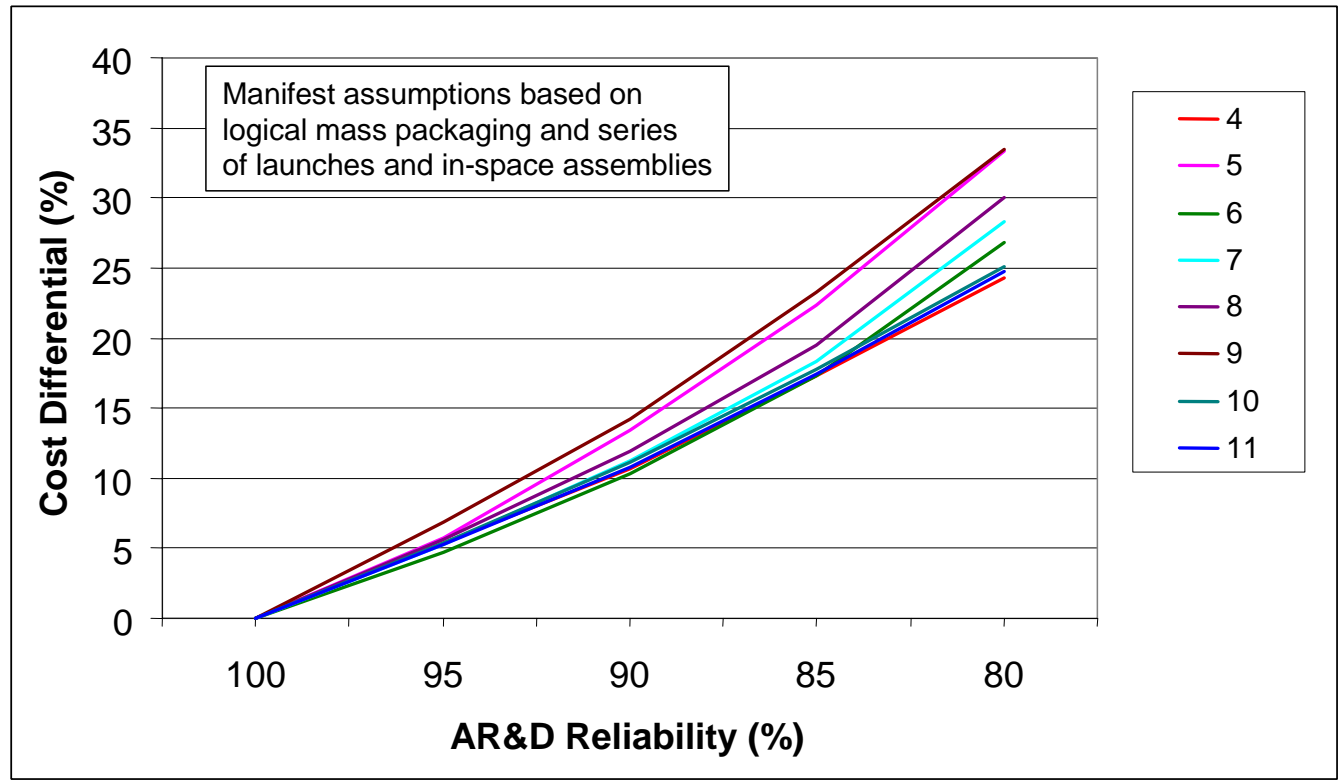

\section{Figure 5. Cost differential for selected cases of modularity having a range of AR\&D reliability for the example reference lunar mission study.}

\section{E. Future Development of DES Model}

Although the Architectural Model captures many of the various logistical operations that need to be analyzed in order to score overall life cycle costs, the model's scope will be expanded in order to address all aspects needed for the Analysis of In-Space Assembly of Modular Systems study. The model currently only simulates operations up through the trans-lunar injection burn, but will need to also simulate trans-lunar operations and transit times, lunar orbit operations, descent operations, surface habitation operations, ascent operations, as well as the logistics involved with returning back to earth. Since the current model only captures launch costs, the various vehicle module costs and mission support costs will also be incorporated. A higher level of fidelity will have to be added to the modeling of on-orbit assembly operations since this is the focal point of the study and the model currently only treats this as a single decision point per module. The distinction being made between robotic and non-robotic interface and assembly operations will be incorporated into this model logic.

The output metrics of the model will be revisited and refined in order to generate metrics that are specific to this study. A scoring function will be generated by the team that takes into account various output parameters from the DES model as well as some from the other discipline tools, which will require that the model be modified to generate the needed metrics.

\section{F. Model Verification and Validation}

In order for any numerical simulation model to be considered credible, some sort of verification and validation (V\&V) methodology is needed (when possible) to ensure that a) the model is correctly coded (verification) and b) the model is modeling the system appropriately (validation). Such "sanity checks" against the model can be accomplished using a wide range of techniques, some quantitative and some qualitative. A particular obstacle that has plagued the application of DES in conceptual design in the space industry is lack of historical numerical data that can be used to corroborate model output data. Current mission objectives pose challenges of going to the Moon that exceed the scope of Apollo, and going to Mars has no known analog. The only analog that comes close to NASA's current exploration goals is the ISS. Because of this, V\&V ends up taking more qualitative forms and relies heavily on expert opinion.

During this study, heavy emphases will be placed on the application of $V \& V$ techniques whenever appropriate to establish "confidence" in the resulting output data. There are many different definitions and listings of various V\&V techniques that can be used to substantiate computer models. One source ${ }^{2}$ in particular lists fourteen different V\&V 
techniques, eight of which can be applied in the case where there is no real-world data to use for corroboration. The eight applicable techniques are as follows:

i.Animation - graphical representation of entity flow throughout the model

ii.Comparison to Other Models - comparison to other life cycle analysis tools in the industry

iii.Degenerate Tests - sanity checks of specific pieces of coding logic

iv.Extreme Condition Tests - analysis of output data resulting from extreme combinations of inputs

v.Face Validity - subject matter expert corroboration of model behavior

vi.Internal Validity - analysis of internal model variability to ensure that model is consistent

vii.Operational Graphics - various performance measures displayed graphically such as queue lengths

viii.Traces - specific entities traced throughout the model to ensure proper logic flow

In addition to these qualitative measures, subject matter experts will analyze all input data and data distributions in order to ensure credible model runs. Part of the purpose of the IDC is to involve discipline experts to allow proper quality control of data being passed between analysis tools. Although it may not be possible to statistically verify and validate computer models of future space operations, particularly one that deals with the on-orbit assembly of modular systems, credibility will be established by SME (Subject Matter Experts) approval of input data in conjunction with the eight qualitative techniques mentioned.

\section{Life Cycle Cost Analysis}

Life Cycle Cost (LCC) analysis is a collective term comprising many kinds of analysis, e.g., reliabilityavailability-maintainability (RAM) analysis, economic analysis, risk analysis, etc. A main objective of the LCC analysis is to quantify the total cost of ownership of a system throughout its full life cycle, which includes research and development, production, operation and maintenance, and disposal. The predicted LCC is useful information for decision making in acquisition strategies, optimizing designs, developing logistics (maintenance) philosophies, or in multi-alternative selections. The Analysis of In-Space Assembly of Modular Systems effort will use LCC analysis as a first line discriminator for underscoring the advantages and disadvantages of competing modularity architectures.

The act of in-space assembly may not necessarily impact the costs in the other areas, shown in Table 1. However, the choices made in those other areas may have strong implications for the costs of launch processing and in-space assembly. For example, as demonstrated above, the number of modules has little impact on the total launch cost for placing those modules in orbit. The reason that launch cost is relatively insensitive to modularity is that the total space exploration system can be cleverly broken down to fit within a variety of launch vehicles having favorable costs. However, breaking the spacecraft into many modules to optimize launch cost may have severe consequences for the costs for in-space assembly, if reliability is affected. For instance, if the spacecraft is broken into 20 launches and the reliability of the selected in-space assembly method is 1 in $20(0.95)$, then the chance of losing one module is "statistically probable". As a result, in the analysis, one (unlucky) module will be lost, and the impact of losing that module will reverberate throughout the entire system of systems and its life cycle. In the analysis, each module will take its turn at being lost, and the sensitivity of the system to its loss will be calculated. Furthermore, if the unlucky module involves the loss of an astronaut, then the costs due to an extensive accident investigation, including an "indefinite" safety stand-down, can be too great for the program to bear. Thus, in-space assembly is highly sensitive to decisions made in other areas. Therefore, requirements for in-space assembly must be fed upstream (to the left in Table 1) so to minimize the likelihood of a "statically probable" and costly incident.

Table 1. Areas of Life Cycle Cost Important to In-Space Assembly of Modular Systems

\begin{tabular}{|c|c|c|c|c|c|}
\hline $\begin{array}{c}\text { Systems } \\
\text { requirements } \\
\text { development }\end{array}$ & $\begin{array}{c}\text { Systems design } \\
\text { \& manufacturing } \\
\text { (modularity } \\
\text { selection) }\end{array}$ & $\begin{array}{c}\text { Flight } \\
\text { certification \& } \\
\text { launch } \\
\text { selection }\end{array}$ & $\begin{array}{c}\text { Launch } \\
\text { processing \& } \\
\text { in-space } \\
\text { assembly } \\
\text { (operations) }\end{array}$ & $\begin{array}{c}\text { Mission } \\
\text { insertion \& } \\
\text { mission } \\
\text { operations } \\
\text { (system of } \\
\text { systems) }\end{array}$ & $\begin{array}{c}\text { System } \\
\text { retirement }\end{array}$ \\
& & & & \\
\hline
\end{tabular}

One overarching constraint to estimating life cycle costs is that one cost modeling technique may not apply to all areas. For instance, cost modeling techniques that are highly sensitive to payload mass may not work well for areas involving an extensive software development and test demonstration project. Software has no mass, yet the cost to develop it can consume the largest part of a project budget. Thus, some care must be given to the selection and sensitivities of the cost modeling used in all areas. Furthermore, some resources may be required to seam the different cost models into one life cycle model. These factors will be explored further for developing the most 
comprehensive yet seamless life cycle costing model possible within the scope of this project. Some guidance is expected from the SBA group during development.

\section{Conclusion}

Current architecture studies illustrate some effects of modularity and the underlying AR\&D reliability on the launch phase of mission life cycle costs. The main goal of Analysis of In-Space Assembly of Modular Systems is to build upon those studies to score competing approaches to assembling and detaching those modules during the entire operational scenario. This project has identified the core experts and fundamental tools necessary to meet that main goal and strives to implement them within a collaborative engineering environment for increased productivity. Once operational, this analysis capability will work in concert with other Advanced Concepts projects and SBA activities to build a coast-to-coast analysis support infrastructure for ESR\&T.

\section{Acronyms}

$\begin{array}{ll}\text { AR\&D } & \text { Autonomous Rendezvous and Docking } \\ \text { ASCT } & \text { Advanced Studies, Concepts, and Tools } \\ \text { CEV } & \text { Crew Exploration Vehicle } \\ \text { DES } & \text { Discrete Event Simulation } \\ \text { DoE } & \text { Design of Experiments } \\ \text { ESR\&T } & \text { Exploration Systems Research and Technology } \\ \text { GSFC } & \text { Goddard Space Flight Center } \\ \text { IDC } & \text { Integrated Design Center } \\ \text { ISS } & \text { International Space Station } \\ \text { JSC } & \text { Johnson Space Center } \\ \text { KSC } & \text { Kennedy Space Center } \\ \text { LaRC } & \text { Langley Research Center } \\ \text { LEO } & \text { Low Earth Orbit } \\ \text { LCC } & \text { Life Cycle Cost } \\ \text { RAM } & \text { Reliability-Availability-Maintainability } \\ \text { SBA } & \text { Simulation Based Acquisition } \\ \text { VBA } & \text { Visual Basic for Applications } \\ \text { V\&V } & \text { Verification and Validation }\end{array}$

\section{Acknowledgments}

The authors would like to express their gratitude to the many former and present contributors and co-workers among the NASA Centers and participating organizations for a rich heritage from which to build NASA's next exploration missions. Their remarkable successes inspire us to reach new heights through difficult challenges.

\section{References}

${ }^{1}$ Sietzen, F., Jr., “From Columbia to Constellation: Crafting a New Space Policy,” Aerospace America, Vol. 42, No. 4, April 2004, pp. 36-43.

${ }^{2}$ Sargent, R. G., "Verification and Validation of Simulation Models," Proceedings of the 2003 Winter Simulation Conference, IEEE, New Orleans, LA, 2003, pp. 41. 